\title{
If time travel to our location is possible, we do not live in a branching universe
}

This paper argues for the following disjunction: either we do not live in a world with a branching temporal structure, or backwards time travel ${ }^{1}$ is nomologically impossible, given the initial state of the universe, or backwards time travel to our space-time location is impossible given large-scale facts about space and time. A fortiori, if backwards time travel to our location is possible, we do not live in a branching universe.

Horwich (1987:1) gestures towards an interesting argument for the conclusion that there is no backwards time travel in the actual world. For, if there is some future time, $t$, at which the requisite technology is developed and subsequently used, we would expect to see, around here, time travellers originating from t. We see no such time travellers, and thus there is no actual time travel. ${ }^{2}$ Call this (following Smith, 2016) the 'where are the time travellers?' argument. Fulmer (1980) articulates, but declines to endorse, the same style of argument. Reinganum (1986) argues in a similar vein that if there is actual time travel then greedy time travellers would seek enormous wealth (by investing money, travelling forward to collect their capital and interest, and repeating this process at their whim). As the havoc this would wreak on our financial system has not ensued, Reinganum concludes that time travel "has never existed and furthermore never will exist" (1986:10).

The 'where are the time travellers?' argument is unconvincing. There are two broad reasons why we might see no time travellers, despite there being actual time travel. The first concerns contingent features of the travellers themselves. Perhaps they have visited, and we have simply not noticed, either because they chose to remain covert or because we have been insufficiently attentive (see Smith, 1997; Fulmer, 1980). Perhaps they have simply not chosen to visit us, for our little corner of space-time is mundane in comparison to the time travel tourist hotspots. Perhaps time travel is a very costly undertaking, and time travellers must be very judicious in their use of it. The second concerns large-scale facts about space and time that might prevent time travellers from

\footnotetext{
${ }^{1}$ When I talk of backwards time travel, I have in mind the interesting kind, where future individuals can travel back and interact with past individuals.

2 "If the necessary technology will ever become available, why haven't we encountered visitors from the future?” (1987:1). In chapter 7 of the same volume, Horwich presents his better-known argument against time travel: namely, that backwards time travel would result in "an endless string of improbable coincidences" (1987:123). This latter argument is given a thorough treatment in Smith (1997), and is not my focus here. Likewise, while Hawking (1992:610) notes that "[t]here is also strong experimental evidence in favor of the conjecture [that there is no actual time travel] from the fact that we have not been invaded by hordes of tourists from the future", his primary argument - that there is no actual time travel due to the nomological impossibility of closed time-like curves-is not my focus.
} 
visiting us. For example, it could be that time travel to our location has not occurred because we are not near to the exit of a naturally existing wormhole, and we have not created such a wormhole (Smith, 2016). In other words, it could be that the earliest destination to which one can time travel is after the time at which we exist.

While my primary focus is the first type of response, let's take a moment to consider the second. This explanation of the lack of observed time travellers derives from contemporary models of how backwards time travel might work—see, e.g., Morris, Thorne and Yurtsever (1988)—which typically suggest that a time traveller would take a 'shortcut' between two space-time locations, via a wormhole. There are three ways to understand how this might work. It could be that:

(a) The only nomologically possible way to time travel is to harness a naturally occurring wormhole that connects two space-time locations.

(b) It is nomologically possible to create (or make traversable) a wormhole in the present, which would allow future time travellers to visit us, but not allow travel before its creation. ${ }^{3}$

(c) It is nomologically possible to create (or make traversable) a wormhole in the present, through which one could travel to the past.

If (a) or (b) is the case, then the 'where are the time travellers?' argument can be dismissed by pointing out that there are no naturally occurring wormhole exits near us, and that we have not created a wormhole from which future time travellers can emerge. In my conclusion, this case is captured by the disjunct that 'backwards time travel to our space-time location is impossible given large-scale facts about space and time'.

If (c) is the case, and wormholes can be created between the traveller's location and a past destination, then there would still need to be a wormhole near us in order for time travellers to arrive. However, we cannot explain the lack of such a wormhole purely in terms of contingent large-scale facts about space and time, or our own failure to create one. That there is no traversable wormhole near us is, in part, due to the fact that no

\footnotetext{
${ }^{3}$ Davies (2007) notes that "[p]ossibly space is threaded with such structures naturally--relics of the big bang. If so, a supercivilization might commandeer one. Alternatively, wormholes might naturally come into existence on tiny scales, the so-called Planck length, about 20 factors of 10 as small as an atomic nucleus. In principle, such a minute wormhole could be stabilized by a pulse of energy and then somehow inflated to usable dimensions."

${ }^{4}$ That's because one large-scale fact about space and time concerns whether or not there is a wormhole exit near us, be it naturally occurring or artificial.
} 
future time travellers have created one. Moreover, as I argue below, if we live in a branching universe and (c) is the case, future time travellers would create and conspicuously emerge from a wormhole near us. ${ }^{5}$ As this is not occurring, I conclude that if (c) is the case, we do not live in a branching universe. ${ }^{6}$

Thus, it is primarily the possibility of (c) that motivates my discussion of the first type of response to the 'where are the time travellers?' argument. (c) has been given little attention in the existing literature, but, as far as I am aware, this is not due to any knockdown argument against it. As worlds with time travel are worlds with backwards causation, on what basis can we rule out that a wormhole in the present might be caused to exist by some future time travellers? In any case, no matter which of (a), (b) or (c) is true, if backwards time travel to our location is possible, we do not live in a branching universe.

I will now argue that the first type of response, that appeals to contingent features of the time travellers, is implausible in the context of the 'branching universe' ontological picture, whereby every way that things could go, given the way things are and the laws of nature, is the case on some branch or other. ${ }^{7}$ First, let's consider the purely B-theoretic incarnation of the view, according to which the entire branching structure exists unchangingly, like the eternalist's four-dimensional block. The model is intended to capture the openness of the future, and is often motivated as the natural way to understand the ontology implicit in the Everettian interpretation of quantum mechanics. The latter motivation lends the view scientific credibility: perhaps the branching universe is not merely a metaphysician's fantasy, but an empirically respectable hypothesis. For discussion of this version see, inter alia, Thomason (1970), Belnap (1992), Farr (2012) and Wilson (manuscript).

Even if there are limitations on the branches that are accessible via time travel in a branching universe, if we live in such a universe it cannot be that we see no time

\footnotetext{
5 Whether there is in fact a local exit from a traversable wormhole is a difficult issue, and one beyond the scope of this paper. Wormholes are, in principle, detectable. Indeed, they are detectable with current technology. Nevertheless, detecting very small wormholes remains very difficult. Since it is unclear how large a wormhole would need to be in order to be traversable it is, in turn, unclear whether it would be easy for us to detect traversable wormholes. Thus, it seems premature to conclude that there are no exits from traversable wormholes near us (though it is clear that, if there are such wormholes, they are not being conspicuously traversed!).

${ }^{6}$ A corollary of this is that if we live in a branching universe, and time travel is nomologically possible given the initial conditions, it works in the way outlined by option (a) or (b).

${ }^{7}$ Notably, this rejoinder is not available to Horwich, who discusses the branching universe but dismisses it as unmotivated (1987:25-33).
} 
travellers merely in virtue of contingent features of the time travellers themselves. To see this, suppose that one can only time travel between two points if there is a direct path between those points (where a direct path is one that only runs forwards or backwards in time; a path that runs back along one branch, and then forward along another is not a direct path). ${ }^{8}$ Nonetheless, looking forward from our location, there is a lot of future from which time travellers could originate. Moreover, that future is very diverse. Just as the branching structure includes every nomologically possible way things could go, compatible with the initial conditions of the universe, the branching structure in our future includes every nomologically possible way things could go, given the way things are now (including, on the quantum version of the branching universe, all the possible but vanishingly unlikely quantum events. See Wilson, (manuscript \$1.8) for a discussion of the plenitude of the quantum many-worlds).

So, if time travel is nomologically possible and compatible with the current state of the world, there are many_perhaps even infinitely many_-branches in our future where time travel technology is developed and used. ${ }^{9}$ Likewise, there will be, amongst these branches, branches where the inhabitants are motivated to travel back and visit our little corner of space-time (not merely to visit 2017-there will be many 2017s within the branching structure-but to visit our 2017). Amongst these many branches containing time travellers itching to visit us, there will be many branches where these individuals are also wild exhibitionists who, upon arriving at our time, intend to present an enormous fanfare to make very sure that we are aware of their arrival. Finally, amongst these branches there will be branches populated by individuals with one eye, two eyes, three eyes, and so on... As all of these things are nomologically possible, they are all out there in the enormous future branching structure.

Given the existence of all these future branches, we can't simply be unlucky enough to inhabit a world with only shy time travellers, or time travellers uninterested in us: a branching universe where there is time travel will contain the multiplicity of branches I

\footnotetext{
${ }^{8}$ The argument that follows is equally effective if we suppose there to be no such limitations on the accessibility relations between branches. I mention these limitations only to show that the argument requires no potentially objectionable assumptions about the possible routes via which time travel might occur in a branching universe.

9 There will be many such branches unless for some reason time travel is so sensitive that it requires, say, a kind of quantum event that can only occur given the conditions that obtain on one branch, but not the nearby branches distinct from it in tiny ways — such as the decaying of atoms at the (spatially) distant end of the universe. But why should we think time travel, if possible, is sensitive in this way? Plausibly, if there is time travel anywhere in the branching structure, there is time travel in many places, including in our future.
} 
described above, exhibitionists and all! Thus, there will be many-perhaps infinitely many-beings who have access to time travel technology, desire to visit us, and are intent on putting on a show when they do so. So, if we live in a branching universe, and time travel is nomologically possible given the initial conditions, and there are no largescale facts about space-time that prevent time travel to our location, we should not merely expect some evidence of time travellers-we should expect to be inundated by them! There should be one-eyed exhibitionists, two-eyed exhibitionists, three-eyed exhibitionists, and so on. ${ }^{10}$

Let's now briefly consider the A-theoretic branching universe model, due to McCall (1994). ${ }^{11}$ According to this view, the objective present 'moves through' the branching structure, such that at each fork only one future path is 'chosen', and the others 'drop off. ${ }^{12}$ As argued by Miller (2005) in the context of a different argument against time travel in an A-theoretic branching universe, if a time traveller arrives from a future branch which subsequently drops off, that individual should no longer be considered a time traveller, for a necessary condition on being a (backwards) time traveller is to be caused by some future event. ${ }^{13}$ If Miller is right, and if time travel to here is nomologically possible, given the initial conditions of the universe and large-scale facts about space-time, we should expect to be inundated by folk who appear to be time travellers. However, unless the branches from which they originate are on the path taken by the objectively present moment, there will, bizarrely, be no actual time travel. Ultimately, whether these individuals deserve to be called time travellers need not concern us here. As a matter of fact, we are experiencing no such inundation, and thus the arguments of the previous paragraph apply equally to the A-theoretic branching universe model.

In sum, if we inhabit a branching universe, the first style of response to the 'where are the time travellers?' argument is implausible. Thus, we arrive at the disjunction with

\footnotetext{
${ }^{10}$ A referee wondered what to say about personal identity in the case where we are inundated by time travelling continuers of ourselves. The natural response is to say that this is a case of massive fission, where the products of fission then travel back. So one's preferred story about personal identity in the case of fission can be imported to make sense of what's happening.

${ }^{11}$ The following argument is only required on the supposition that Keller and Nelson (2001), Daniels (2012) and Hall (2014) are right that the A-theory is compatible with time travel. If it turns out that, given the A-theoretic picture, there are independent grounds to think that time travel (or perhaps backwards causation, more generally) is impossible or incoherent, the arguments that follow are superfluous. Either way, the disjunctive conclusion holds. Thanks to an anonymous referee for pointing this out.

12 Alternatively, one might think of the A-theoretic version of the view as one where there is an objectively present moment that moves through the atemporally existing four-dimensional structure. The same arguments apply to this view as to the purely B-theoretic view.

${ }^{13}$ See Martínez (2011) for the view that they never were time travellers.
} 
which we started the paper: either we do not live in a world with a branching temporal structure, or backwards time travel is nomologically impossible, given the initial state of the universe, or backwards time travel to our location is impossible given large-scale facts about space and time (such as the lack of a wormhole exit near us). It follows that if backwards time travel to our location is possible, we do not live in a branching universe.

\section{References}

Belnap, N., 1992, "Branching space-time” Synthese, 92(3):385-434.

Daniels, P.R., 2012, "Back to the Present: Defending Presentist Time Travel”, Disputatio, 4(33):469-484.

Davis, P., 2007, "How to build a time machine" Scientific American. https://www.scientificamerican.com/article/how-to-build-a-time-machine-200704/

Farr, M., 2012, "On A- and B-theoretic elements of branching spacetimes” Synthese, 188(1):85-116.

Fulmer, G., 1980, “Understanding time travel”, Southwestern Journal of Philosophy, 11:151-6.

Hall, T., 2014, "In Defense of the Compossibility of Presentism and Time Travel" Logos \& Episteme, 5(2):141-159.

Hawking, S.W., 1992, "Chronology protection conjecture”, Physical Review D, 46: 603-11.

Horwich, P., 1987, Asymmetries in Time: Problems in the Philosophy of Science, Cambridge MA: MIT Press.

Keller, S., \& Nelson, M., 2001, "Presentists should believe in time-travel”, Australasian Journal of Philosophy, 79(3), 333-345.

Martínez, M., 2011, “Travelling in Branching Time”, Disputatio, 4 (31):59-75.

McCall, S., 1994, A Model of the Universe Space-Time, Probability, and Decision. OUP.

Miller, K., 2005, “Time travel and the open future”, Disputatio 1(19):223-32.

Morris, M.S., Thorne, K.S., and Yurtsever, U., 1988, "Wormholes, Time Machines, and the Weak Energy Condition”, Physical Review Letters, 61(13):1446-1449.

Reinganum, M.R., 1986, “Is time travel impossible? A financial proof”, Journal of Porffolio Management, 13:10-2.

Smith, N.J.J., 1997, "Bananas enough for time travel?”, British Journal for the Philosophy of Science, 48:363-89. 
Smith, N.J.J., 2016, “Time Travel”, The Stanford Encyclopedia of Philosophy, Edward N. Zalta (ed.), URL = <https://plato.stanford.edu/archives/spr2016/entries/timetravel $/>$.

Thomason, R., 1970, “Indeterminist time and truth-value gaps”, Theoria, 36(3):264-281.

Wilson, A., manuscript, “The Nature of Contingency: Quantum Mechanics as Modal Realism" 